\title{
RCS: A Reference Model Architecture for Intelligent Control
}

\author{
James S. Albus \\ albusecme.nist.gov \\ William G. Rippey \\ rippey@cme.nist.gov \\ Robot Systems Division, Manufacturing Engineering Laboratory \\ National Institute of Standards and Technology \\ Gaithersburg, MD 20899 USA
}

\begin{abstract}
The Real-time Control System (RCS) is a reference model architecture for intelligent realtime control systems. It partitions the control problem into four basic elements: task decomposition, world modeling, sensory processing, and value judgment. It clusters these elements into computational nodes that have responsibility for specific subsystems, and arranges these nodes in hierarchical layers such that each layer has characteristicfunctionality and timing. The RCS architecture has a systematic regularity, and recursive structure that suggests a canonical form.

Systems based on the RCS architecture have been implemented more or less for a wide variety of applications that include loading and unloading of parts and tools in machine tools, controlling machining workstations, performing robotic deburring and chamfering, and controlling space station telerobots, multiple autonomous undersea vehicles, unmanned land vehicles, coal mining automation systems, postal service mail handling systems, and submarine operational automation systems. Software developers accustomed to using RCS for building control systems have found it provides a structured design approach that makes it possible to reuse a great deal of software.
\end{abstract}

\section{Keywords}

AUTONOMOUS SYSTEMS - CANONICAL MODEL - ENGINEERING METHODOLOGY - HIERARCHICAL CONTROL - INTELLIGENT CONTROL - REAL-TTME CONTROL - REFERENCE ARCHITECTURE - WORLD MODEL

\section{Introduction}

The Real-time Control System (RCS) is a reference model architecture for intelligent real-time control systems. It partitions the control problem into four basic elements: task decomposition, world modeling, sensory processing, and value judgment. It clusters these elements into computational nodes that have responsibility for specific subsystems, and arranges these nodes in hierarchical layers such that each layer has characteristic functionality and timing. The RCS architecture bas a systematic regularity, and recursive structure that suggests a canonical form.

An RCS design paradigm begins with analysis of tasks and goals, then develops control algorithms, data structures, and sensory information necessary to perform the tasks and accomplish the goals. Attempts are underway to develop this approach into a formal methodology for engineering real-time intelligent control systems and analyzing their performance. 
Critics of RCS have objected to its rigidity, i.e. "it forces a system to be built in a certain structure, even if it can be built more effectively in a different architecture". This, however, misses the point of a reference model architecture. A reference model architecture is a canonical form, not a system design specification. Reference models can be implemented in a variety of ways. For example, RCS could be implemented with neural nets (at least in principle). Many of the lower level features can be implemented by finite-state-machines and motion controllers. Higher level functions can be implemented by expert systems, LISP machines, transputers, connection machines, or special purpose processing engines. RCS combines real-time motion planning and control with high level task planning, problem solving, world modeling, recursive state estimation, tactile and visual image processing, and acoustic signature analysis. In fact, the evolution of the RCS concept has been driven by an effort to include the best properties and capabilities of most, if not all, of the intelligent control systems currently known in the literature, from subsumption to SOAR [Laird], from blackboards to object-oriented programming.

By no means, should the apparent completeness of the canonical form of RCS be taken to mean that all of the problems are solved in each of these areas, and that all the issues are resolved-far from it. The enormous complexity of the RCS architecture should lead one to appreciate how difficult the task of creating intelligent systems is going to be. Not so long ago, respected people were predicting that intelligent robots would be able to duplicate human performance before the end of this century. A correct understanding of RCS should lead one to realize that the creation of intelligence may be more complex and scientifically challenging than controlling fusion or analyzing the genome, and possibly more rewarding as well.

This paper is divided into seven sections. Section 1 describes the evolution of the RCS system through its various versions. Section 2 describes the timing of real-time task decomposition (planning and execution) and sensory processing (sampling and integration) at the various layers in the RCS hierarchy. Sections 3, 4, 5, and 6 define the functionality and contents of the Task Decomposition, World Modeling, Sensory Processing, and Value Judgment modules respectively. An Appendix gives an example of RCS applied to a machining workstation application.

\section{Evolution of RCS}

RCS has evolved through variety of versions over a number of years as understanding of the complexity and sophistication of intelligent behavior has increased. The first implementation, RCS-1, was designed for sensory-interactive robotics by Barbera in the mid 1970's [Barbera]. The next generation, RCS-2, was developed by Barbera, Fitzgerald, Kent, and others [Barbera84] for manufacturing control in the NIST Automated Manufacturing Research Facility (AMRF) during the early 1980's [Simpson,Albus82]. The control system for the Army Field Material Handling Robot (FMR)[McCain] was also implemented in RCS-2, as were the Army TMAP and TEAM semi-autonomous land vehicle projects. RCS-3 was designed for the NBS/DARPA Multiple Autonomous Undersea Vehicle (MAUV) project [Albus88] and was adapted for the NASA/NBS Standard Reference Model Telerobot Control System Architecture (NASREM) being used on the space station Flight Telerobotic Servicer [Albus89]. The basic building block of RCS-3 is shown in Figure 1(a). The principle new features introduced in RCS-3 are the World Model and the operator interface. The inclusion of the World Model provides the basis for task planning and for model-based sensory processing. This led to refinement of the task decomposition (TD) modules so that each have a job assigner, and planner and executor for each of the subsystems assigned a job. This corresponds roughly to Saridis' three level control 
hierarchy [Saridis].

RCS-4 is under current development by the NIST Robot Systems Division. The basic building block is shown in Figure 1(b). The principle new feature in RCS-4 is the explicit representation of the Value Judgment (VJ) system. RCS -4 also renames the task decomposition (TD) module, calling it the behavior generating (BG) module. VJ modules provide to the RCS-4 control system the type of functions provided to the biological brain by the limbic system. The VJ modules contain processes that compute cost, benefit, and risk of planned actions; and that place value on objects, materials, territory, situations, events, and outcomes. Value state-variables define what goals are important, and what objects or regions should be attended-to, attacked, defended, assisted, or otherwise acted upon. Value judgments, or evaluation functions, are an essential part of any form of planning or learning. However, the evaluation functions are usually not made explicit, or assigned to a separate module as in RCS -4 . The application of value judgments to intelligent control systems has been addressed by George Pugh [Pugh]. The structure and function of VJ modules are developed more completely in [Albus91].

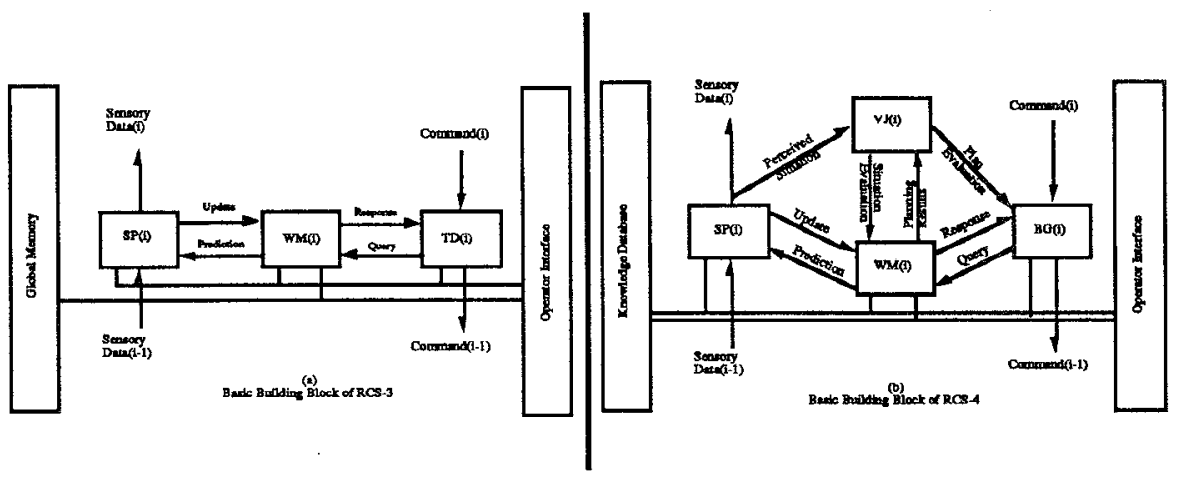

Figure 1. The basic building blocks of the RCS control paradigm.

RCS-4 is designed to address highly autonomous applications in unstructured environments where high bandwidth communications are impossible, such as unmanned vehicles operating on the battlefield, deep undersea, or on distant planets. These applications require autonomous value judgments and sophisticated real-time perceptual capabilities. RCS-3 will continue to be used for less demanding applications such as manufacturing, construction, or telerobotics for near-space, or shallow undersea operations, where environments are more structured and communication bandwidth to a human interface is less restricted.

\section{Organization and Timing in the RCS Hierarchy}

Figure 2 summarizes the relationship in RCS-4 between the organizational hierarchy that is defined by the command tree, the computational hierarchy that is defined along each chain of command, and the behavioral hierarchy that is produced in state-space as a function of time. The behavioral hierarchy consists of state/time trajectories that are produced by computational modules executing tasks in real-time. 
Levels in the RCS command hierarchy are defined by temporal and spatial decomposition of goals and tasks into levels of resolution, as well as by spatial and temporal integration of sensory data into levels of aggregation. Temporal resolution is manifested in terms of loop bandwidth, sampling rate, and state-change intervals. Temporal span is measured in length of historical traces and planning horizons. Spatial resolution is manifested in the resolution of maps and grouping of elements in subsystems. Spatial span is measured in range of maps and the span of control.

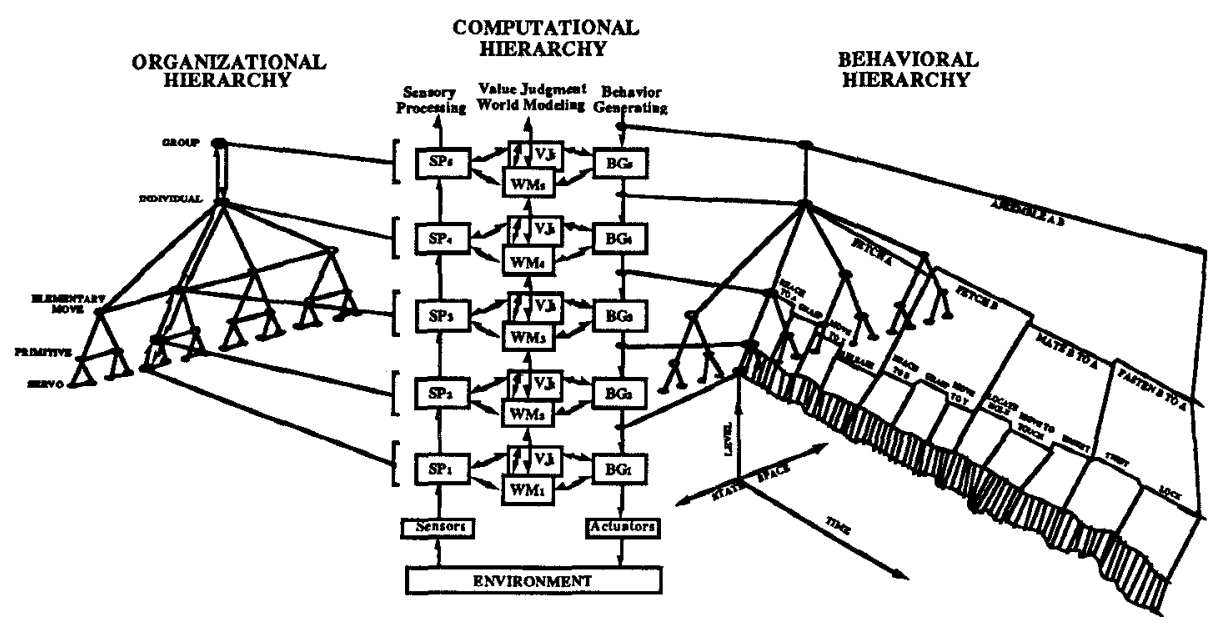

Figure 2. The relationship in RCS-4 between the organizational hierarchy of subsystems, the computational hierarchy of computing modules, and behavioral hierarchy of state trajectories that result as the computing modules execute tasks.

A fundamental principle of RCS-3 and 4 is that planning goes on simultaneously and continuously at all levels. At each level, planners periodically generate plans containing, on average, about ten steps. At each successive level, the first one or two steps in the current plan from the level above are further decomposed into subplans with an order of magnitude more resolution, and an order of magnitude less span in time and spacc. As a result, the average period between task commands decreases by about an order of magnitude at each lower level.

Replanning is done either at cyclic intervals, or whenever emergency conditions arise. The cyclic replanning interval may be as short as the average command update interval at each level, or about about ten percent of the planning horizon at each level. This allows the real-time planner to generate a new plan about as often as the executor generates a new output command. Less frequent replanning will reduce the computational speed requirements for real-time planning, but may also reduce control system effectiveness. Emergency replanning begins immediately upon the detection of an emergency condition.

Task execution also goes on simultancously and continuously at all levels. At each level, executors periodically sample feedback, compare it with the current plan, and issue output commands to correct for deviations between plans and observations. At each sampling period feedback is tested for emergency conditions, and preplanned emergency recovery routines are invoked immediately upon detection of any emergency condition. This allows the system to 
react to emergencies within a single control cycle with preplanned recovery routines that bridge the temporal gap between when an emergency is detected and when the appropriate planners have new plans ready for execution.

A duality exists between the task decomposition and the sensory processing hierarchies. At each hierarchical level a task can be decomposed into a set of subtasks at the next lower level. At each level a sensory event can be composed from a sequence of events at the next lower level, a recognized entity can be composed from a group of subentities at the next lower level. At each level, the sensory processing modules look back into the past about as far the planner modules look forward into the future. At each level, future plans have about the same detail as historical traces.

\section{Task Decomposition}

Each task decomposition (TD) module at each level consists of three sublevels: Job Assignment, Planning, and Execution.

The job assignment (JA) submodule is responsible for spatial task decomposition. It partitions the input task command into $\mathrm{N}$ spatially distinct jobs to be performed by $\mathrm{N}$ physically distinct subsystems, where $\mathrm{N}$ is the number of subsystems currently assigned to the TD module. The JA submodule is also responsible for assigning tools and allocating physical resources (such as robotic arms and hands, sensors, tools, and materials) to each of its subordinate subsystems for their use in performing their assigned jobs.

For each of the $\mathrm{N}$ subsystems, there exists a planner submodule PL(j). Each planner submodule is responsible for decomposing the job assigned to its subsystem into a temporal sequence of planned subtasks. Each planner submodule is also responsible for resolving conflicts and mutual constraints between hypothesized plans of submodules.

There is an executor EX(j) for each planner PL(j). The executor submodules are responsible for successfully executing the plan state-graphs generated by their respective planners. When an executor is informed by the world model that a subtask in its current plan is successfully completed, the executor steps to the next subtask in that plan. When all the subtasks in the current plan are successfully executed, the executor steps to the first subtask in the next plan. If the feedback indicates the failure of a planned subtask, the executor branches immediately to a preplanned emergency subtask. Its planner simultaneously begins work selecting or generating an error recovery sequence which can be substituted for the former plan which failed. Output subcommands produced by executors at level $\mathrm{i}$ become input commands to job assignment submodules in TD modules at level i-1.

Planners PL(j) constantly operate in the future, each generating a plan to the end of its planning horizon. The executors EX(j) always operate in the present, at time $t=0$, constantly monitoring the current state of the world reported by feedback from the world model.

At each level, each executor submodule closes a reflex arc, or servo loop, and the executor submodules at the various hierarchical levels form a set of nested servo loops. The executor loop bandwidth decreases about an order of magnitude at each higher level. Each level has a dominant frequency of execution. The actual frequency is dictated by the physical equipment and the application. 


\section{World Modeling}

The world model is an intelligent system's internal representation of the external world. It is the system's best estimate of objective reality. It acts as a buffer between sensory processing and task decomposition. This enables the task decomposition system to act on information that may not be immediately or directly observable by sensors, and enables the sensory processing system to do recursive estimation based on a number of past sensory measurements. The world model is hierarchically structured and distributed such that there is a world model (WM) module with knowledge database (KD) in each node at every level of the RCS control hierarchy. The KD forms a passive, hierarchically structured, distributed data store. The WM provides the operations that act upon the KD. At each level, the WM and value judgment (VJ) modules perform the functions illustrated in Figure 3.

\section{The Knowledge Database}

The world model knowledge database (KD) includes both apriori information, which is available to the intelligent system before action begins, and aposteriori knowledge, which is gained from sensing the environment as action proceeds. The $\mathrm{KD}$ represents information about space, time, entities, events, states of the world, and laws of nature. Knowledge about space is represented in maps. Knowledge about entities, events, and states is represented in lists and frames. Knowledge about the laws of physics, chemistry, optics, and the rules of logic and mathematics is represented in the WM functions that generate predictions and simulate results of hypothetical actions. Laws of nature may be represented as formulae, or as IF/THEN rules of what happens under certain situations, such as when things are pushed, thrown, or dropped.

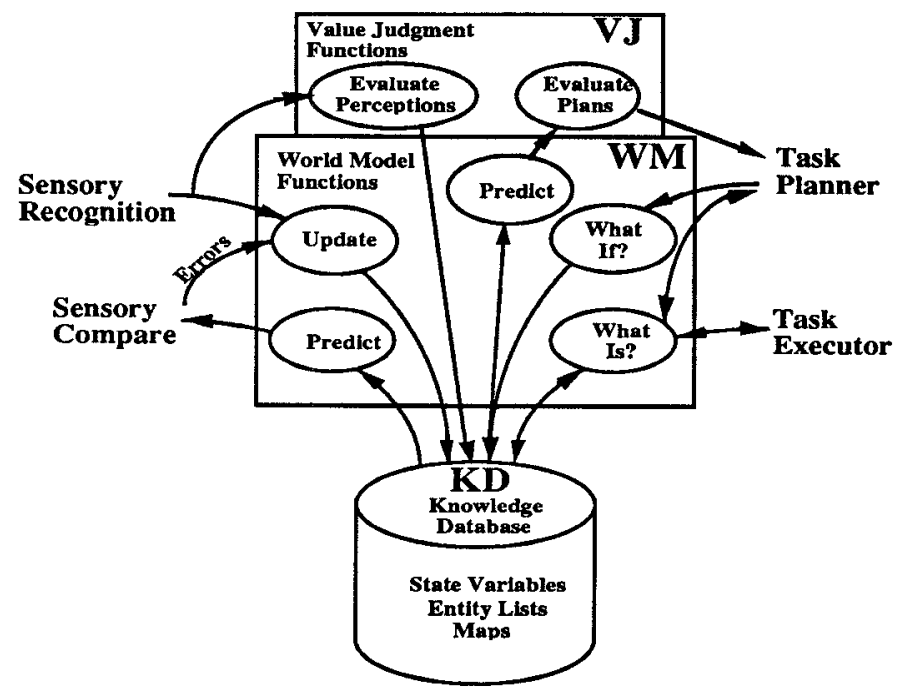

Figure 3. Functions performed by the WM module. 1) Update the knowledge database with recognized entities. 2) Predict sensory data. 3) Answer "What is?" queries from task executor and return current state of world. 4) Answer"What if?" queries from task planner and predict results for evaluation. 
The world model also includes knowledge about the intelligent system itself, such as the values assigned to goal priorities, attribute values assigned to objects, and events; parameters defining kinematic and dynamic models of robot arms or machine tool stages; state variables describing internal pressure, temperature, clocks, fuel levels, body fluid chemistry; the state of all the currently executing processes in each of the TD, SP, WM, and VJ modules; etc.

The correctness and consistency of world model knowledge is verified by sensors and sensory processing SP mechanisms that measure differences between world model predictions and sensory observations. These differences may be used by recursive estimation algorithms to keep the world model state variables the best estimates of the state of the world. Attention algorithms may be used to limit the number of state variables that must be kept up-to-date and any one time.

\section{Sensory Processing}

Sensory processing is the mechanism of perception. Perception is the establishment and maintenance of correspondence between the intemal world model and the external real world. The function of sensory processing is to extract information about entities, events, states, and relationships in the external world, so as keep the world model accurate and up to date.

The Sensory Processing (SP) modules are responsible for gathering data from sensors, filtering and integrating this data, and interpreting it. Noise rejection techniques such as Kalman filtering are implemented here, as well as feature extraction, pattern recognition, and image understanding. At the upper levels of the hierarchy, more abstract interpretations of sensory data are performed and inputs from a variety of sensor systems are integrated over space and time into a single interpretation of the the external world.

Each SP module at each level consists of five types of operations: Coordinate transformation, Comparison, Temporal Integration, Spatial Integration, and Recognition/Detection, as shown in Figure 4.

It has long been known that biological sensory processing occurs in a hierarchy of processing modules, and that perception proceeds by "chunking," i.e. by recognizing patterns, groups, strings, or clusters of points at one level as a single feature, or point in a higher level, more abstract space. It also has been observed that this chunking process proceeds by about an order of magnitude per level, both spatially and temporally [Miller, Meystel]. Thus, at each level in the proposed architecture, SP modules integrate, or chunk, information over space and time by about an order of magnitude.

\section{Value Judgments}

Value judgments provide the criteria for making intelligent choices. Value judgments evaluate the costs, risks, and benefits of plans and actions, and the desirability, attractiveness, and uncertainty of objects and events. Value judgment modules produce evaluations that can be represented as value state-variables. Value state-variables can thus label objects, situations, or places as good or bad, friendly or hostile, attractive or repulsive.

Value judgment algorithms may evaluate risk and compute the level of uncertainty in the recognition of entities and the detection of events. Knowledge in the world model can thus be labeled as reliable or uncertain.

Value judgments can evaluate events as important or trivial. The utilization of memory can be optimized by storing only those events and situations evaluated as "important". Those events labeled as "trivial" can safely be forgotten and cleared from memory. Intelligent machines that 
include capabilities for leaming require value judgment functions to indicate what to "reward" and what to "punish."

Cost/benefit value judgment algorithms can be used to evaluate plans or steer task execution so as to minimize cost and maximize benefits. Value state-variables can be assigned to the attribute lists of plans and actions in task frames to label tasks and plans as good or bad, costly or inexpensive, risky or safe, with high or low expected payoff. Priorities are value state-variables that provide estimates of importance. Priorities can be assigned to task frames so that planners and executors can decide what to do first, how much effort to spend, how much risk is prudent, and how much cost is acceptable, for each task. Value state-variables can thus be used by the behavior generation modules both for planning and executing actions. They provide criteria for making decisions about which course of action to take. Priorities may also determine the degree of alertness, or tactics chosen for planning tasks in a hostile environment. A priority on safety may produce conservative tactics. A priority on aggression may produce an attack on an enemy. Such priorities are typically input from a human commander.

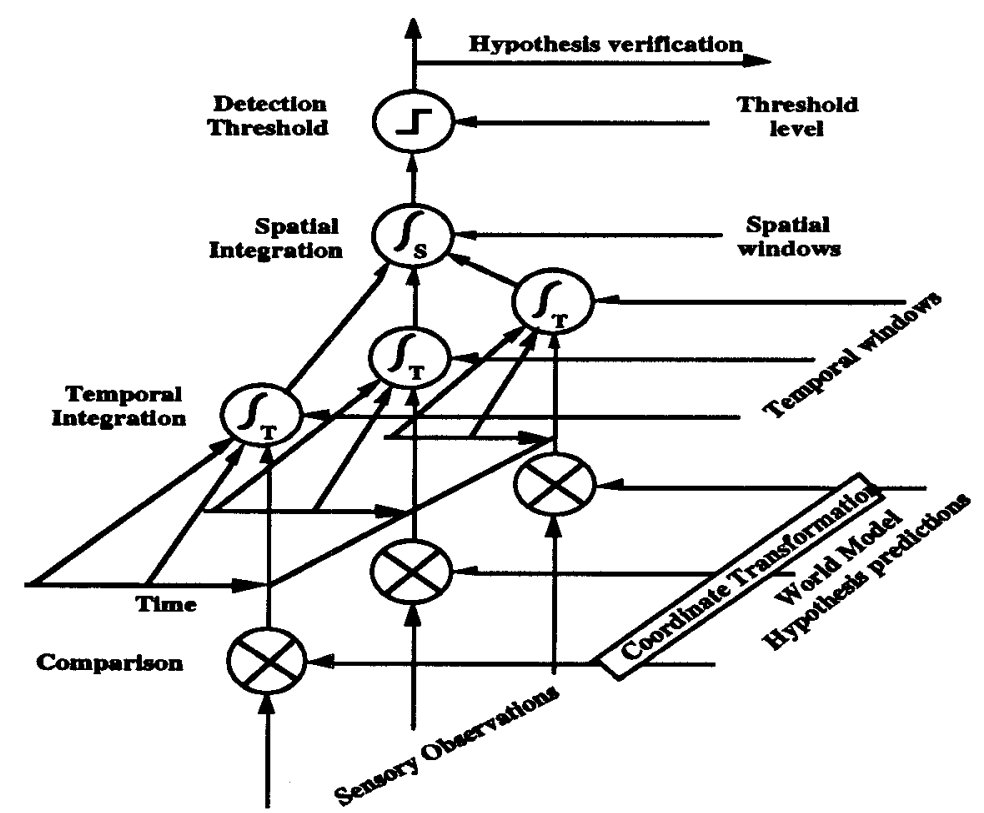

Figure 4. Each sensory processing SP module consists of: 1) a set of coordinate transformers, 2) a set of comparators that compare sensory observations with world model predictions, 3 ) a set of temporal integrators that integrate similarities and differences, 4) a set of spatial integrators that fuse information from different sensory data streams, and 5) a set of threshold detectors that recognize entities and detect events.

Intelligent systems designed for military purposes typically require value judgments labeling objects in the world model as "friend" or "foe." If an object is labeled "friend" it should be defended or assisted. If it is labeled "foe" it should be attacked or avoided. The computation of "friend or foe" may be accomplished by signal analysis or pattern recognition. This computation 
may be very difficult, or trivially simple. For example, in current battlefield situations, friendly systems typically carry transponders that actively emit signals, or modulate reflected energy in a distinctive way that makes recognition of "friend" easy, even in smoke or fog or at night. Often, anything else is assumed to be "foe".

Value state-variables may be assigned by a human programmer or operator, or they may be computed by value judgment functions residing in VJ modules. Inputs to VJ modules describe entities, events, situations, and states. Inputs may also include sensor signal-to-noise ratio, variance between sensory observations and world model predictions, degree of success in goal achievement, and reward or punishment signals from a variety of sources. VJ value judgment functions may compute measures of cost, risk, and benefit. VJ outputs are value state-variables that may be assigned to objects, events, or plans.

\section{Conclusion}

RCS is a reference model architecture that defines what types of functions are required in a realtime intelligent control system, and how these functions are related to each other. It is not a system design or a specification of how to implement specific systems. RCShas, nevertheless, been found useful by many researchers and engineers for designing intelligent machine systems.

The RCS reference model architecture has been developed over a number of years by a number of different researchers. It has evolved through several versions, and has been applied to many applications. It incorporates ideas from many different fields and many different research laboratories. It represents a conscious effort to pull together concepts from the fields of neurosciences, machine intelligence, and control, and to include the best ideas from most of the current researchers in these fields. This, of course, is impossible to do even in a paper much longer than this. Even to list all the relevant references would require many pages. As a result many important issues have been glossed over, or trivialized, and much important work is not referenced. A more complete, but by no means exhaustive bibliography is given in [Albus91].

The reader should not assume that all, or even most, of the elements of the RCS architecture have been fully implemented, or even could be implemented, given the current state of knowledge and technology. Many difficult and subtle problems remain unsolved in task planning and execution, world modeling, communication, knowledge representation, and sensory processing, and many years of research are still needed. Most of the issues related to value judgments have hardly even been addressed.

Current NIST research is directed towards understanding how to use the RCS reference model as a design tool, and how to define an engineering methodology for implementing real-time intelligent control systems based on the RCS paradigm.

\section{References}

[Laird] J.E. Laird, A. Newell, and P. Rosenbloom, "SOAR: An Architecture for General Intelligence", Artificial Intelligence, vol. 33, 1987, pp. 1-64.

[Barbera79] Barbera, A.J., J.S. Albus, M.L. Fitzgerald, "Hierarchical Control of Robots Using Microcomputers," Proceedings of the 9th International Symposium on Industrial Robots, Washington, DC, March 1979.

[Albus71] J.S. Albus, “A Theory of Cerebellar Function”, Mathematical Biosciences, Vol. 10, pgs. 25-61, 1971. 
[Albus75] J.S. Albus, “A New Approach to Manipulator Control : The Cerebellar Model Articulation Controller (CMAC)", Transactions ASME, September 1975.

[Tinbergen] N. Tinbergen, The Study of Instinct, Clarendon, Oxford, 1951.

[Brooks] R. Brooks, "A Robust Layered Control System for a Mobile Robot", IEEE Journal of Robotics and Automation, vol. RA-2, 1, March, 1986.

[Barbera84] A.J. Barbera., J.S. Albus, M.L. Fitzgerald, and L.S. Haynes, "RCS: The NBS RealTime Control System," Robots 8 Conference and Exposition, Detroit, MI, June 1984.

[Simpson] J.A. Simpson, R.J. Hocken, and J.S. Albus, "The Automated Manufacturing Research Facility of the National Bureau of Standards", Journal of Manufacturing Systems, Vol. 1, No. 1, 1983.

[Albus82] J.S. Albus, C. McLean, A.J. Barbera, and M.L. Fitzgerald, "An Architecture for RealTime Sensory-Interactive Control of Robots in a Manufacturing Environment", 4th IFAC/IFIP Symposium on Information Control Prablems in Manufacturing Technology, Gaithersburg, MD, October 1982.

[McCain] H.G. McCain, R.D. Kilmer, S. Szabo, A. Abrishamian, "A Hierarchically Controlled Autonomous Robot for Heavy Payload Military Field Applications,"Proceedings of the International Conference on Intelligent Autonomous Systems, Amsterdam, The Netherlands, December 8-11, 1986.

[Albus88] J.S. Albus, "System Description and Design Architecture for Multiple Autonomous Undersea Vehicles", National Institute of Standards and Technology, Technical Report 1251, Gaithersburg, MD, September 1988.

[Albus89] J.S. Albus, H.G. McCain, and R. Lumia, "NASA/NBS Standard Reference Model for Telerobot Control System Architecture (NASREM)," National Institute of Standards and Technology, Technical Report 1235, Gaithersburg, MD, April 1989.

[Saridis] G.N. Saridis, "Foundations of the Theory of Intelligent Controls", IEEE Workshop on Intelligent Control, 1985.

[Albus91] J.S. Albus, “Outline for a Theory of Intelligence”, IEEE Trans. on Systems, Man, and Cybemetics, Vol.21, No.3, May/June 1991.

[Kelmar] L.R. Kelmar, R. Lumia, "World Modeling for Sensory Interactive Trajectory Generation," Proceedings of the Third International Symposium on Robotics and Manufacturing, Vancouver, BC, Canada, July 18-20, 1990.

[Miller] G. A. Miller, "The Magical Number Seven, Plus or Minus Two: Some Limits on Our Capacity for Processing Information", The Psychological Review, 63, pp.71-97, 1956.

[Meystel] A. Meystel, "Theoretical Foundations of Planning and Navigation for Autonomous Robots", International Journal of Intelligent Systems, 2. 73-128, 1987.

[Pugh] G.E. Pugh and G.L. Lucas, "Applications of Value-Driven Decision Theory to the Control and Coordination of Advanced Tactical Air Control Systems", DecisionScience Applications, Inc., Report No. 218, April 1980.

\section{Appendix: A Machining Workstation Example}

Figure 5 illustrates how the RCS-3 system architecture can be applied to a specific machining workstation consisting of a machine tool, a robot, and a part buffer. RCS-3 produces a layered graph of processing nodes, each of which contains a Task Decomposition (TD), World Modeling (WM), and Sensory Processing (SP) module. These modules are richly interconnected to each other by a communications system. The global memory, or knowledge database, and operator interface are not shown in Figure 5. 
Figure 5 illustrates the ability of RCS-3 to integrate discrete sensors such as microswitches with more complex sensors such as cameras and resolvers. Discrete commands can be issued to valves and fixtures, while continuous signals are provided to servoed actuators.

The branching of the control tree (for example, between the camera and manipulator subsystems of the robot), may depend on the particular algorithm chosen for decomposing a particular task. The specifications for branching reside in the task frame of the current task being executed. Similarly, the specification for sharing information between WM modules at a level also are task dependent. In Figure 5, the horizontal curved lines represent the sharing of state information between subtrees in order to synchronize related tasks. The information that must be shared is also dependent on the specific choice of task decomposition algorithms defined in the task frame.

The functionality of each level in the control hierarchy can be derived from the characteristic timing of that level, and vice versa. For example, in a manufacturing environment, the following hierarchical levels are becoming more or less standard.

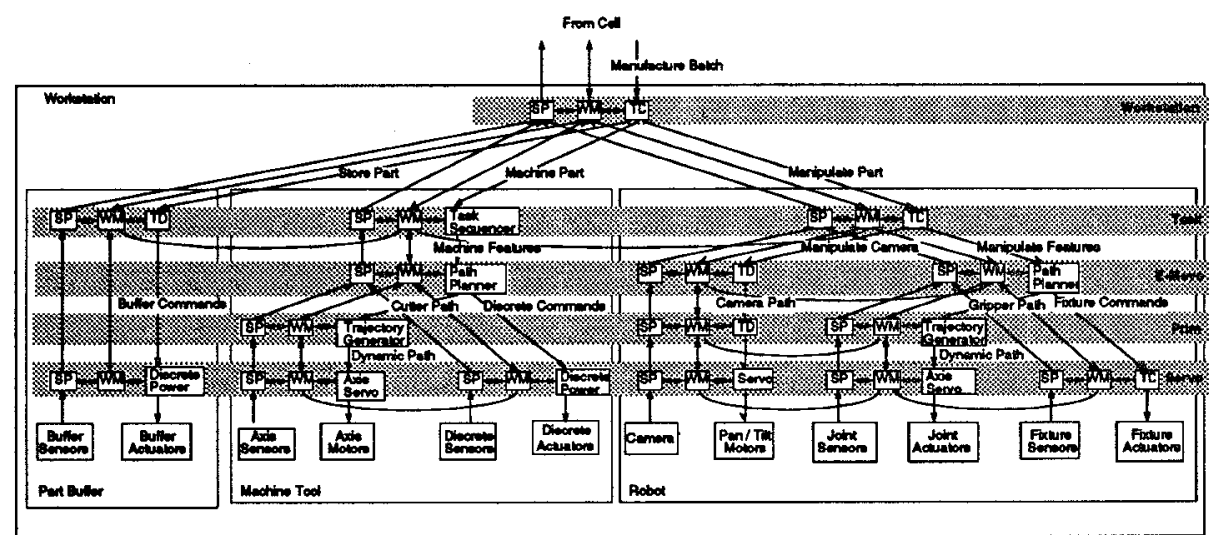

Figure 5. A RCS-3 implementation of a typical machining workstation. The groupings of SP, WM, and TD modules represent computing nodes. In some cases, the functionality of a TD module (e.g. Path Planner, Trajectory Generator, Axis Servo, etc.) is substituted for its generic name.

Level 7-Shop

The shop level schedules and controls the activities of one or more manufacturing cells for an extended period on the order of 24 hours. (The specific timing numbers given in this example are representative only, and may vary from application to application.) At the shop level, orders are sorted into batches and commands are issued to the cell level to develop a production schedule for each batch. At the shop level, the world model symbolic database contains names and attributes of orders and the inventory of tools and materials required to fill them. Maps describe the location of, and routing between, manufacturing cells.

Level 6-Cell

The cell level schedules and controls the activities of several workstations for about a one hour look ahead. Batches of parts and tools are scheduled into workstations, and commands are issued to workstations to perform machining, inspection, or material handling operations on batches or trays of parts. The world model symbolic database contains names and attributes of batches of parts and the tools and materials necessary to manufacture them. Maps describe the location of, and routing between, workstations. (The Shop and Cell levels are above the levels 
shown in the Figure 5 example.) The output from the cell level provides input to the workstation level.

Level 5-Workstation

The workstation level schedules tasks and controls the activities within each workstation with about a five minute planning horizon. A workstation may consist of a group of machines, such as one or more closely coupled machine tools, robots, inspection machines, materials transport devices, and part and tool buffers. Plans are developed and commands are issued to equipment to operate on material, tools, and fixtures in order to produce parts. The world model symbolic database contains names and attributes of parts, tools, and buffer trays in the workstation. Maps describe the location of parts, tools, and buffer trays.

Level 4 -Equipment task

The equipment level schedules tasks and controls the activities of each machine within a workstation with about a 30 second planning horizon. (Tasks that take much longer may be broken into several 30 second segments at the workstation level.) Level 4 decomposes each equipment task into elemental moves for the subsystems that make up each piece of equipment. Plans are developed that sequence elemental movements of tools and grippers, and commands are issued to move tools and grippers so as to approach, grasp, move, insert, cut, drill, mill, or measure parts. The world model symbolic database contains names and attributes of parts, such as their size and shape (dimensions and tolerances) and material characteristics (mass, color, hardness, etc.). Maps consist of drawings that illustrate part shape and the relative positions of part features.

Level 3-Elemental move (E-move)

The E-move level schedules and controls simple machine motions requiring a few seconds, such GO-ALONG-PATH, MOVE-TO-POINT, MILL-FACE, DRILL-HOLE, MEASURESURFACE, etc. (Motions that require significantly more time may be broken up at the task level into several elemental moves.) Plans are developed and commands are issued that define safe path waypoints for tools, manipulators, and inspection probes so as to avoid collisions and singularities, and assure part quality and process safety. The world model symbolic database contains names and attributes of part features such as surfaces, holes, pockets, grooves, threads, chamfers, burrs, etc. Maps consist of drawings that illustrate feature shape and the relative positions of feature boundaries.

Level 2-Primitive

The primitive level plans paths for tools, manipulators, and inspection probes so as to minimize time and optimize performance. It computes tool or gripper acceleration and deceleration profiles taking into consideration dynamical interaction between mass, stiffness, force, and time. Planning horizons are on the order of $\mathbf{3 0 0}$ milliseconds. The world model symbolic database contains names and attributes of linear features such as lines, trajectory segments, and vertices. Maps (when they exist) consist of perspective projections of linear features such as edges, lines or of tool or end-effector trajectories.

Level l-Servo level

The servo level transforms commands from tool path to joint actuator coordinates. Planners interpolate between primitive trajectory points with a 30 millisecond look ahead. Executors servo individual actuators and motors to the interpolated trajectories. Position, velocity, or force servoing may be implemented, and in various combinations. Commands that define actuator torque or power are output every 3 milliseconds (or whatever rate is dictated by the machine dynamics and servo performance requirements). The servo level also controls the output drive signals to discrete actuators such as relays and solenoids. The world model symbolic database contains values of state variables such as joint positions, velocities, and forces, proximity sensor readings, position of discrete switches, condition of touch probes, as well as image attributes associated with camera pixels. Maps consist of camera images and displays of sensor readings. 